\title{
CONTRASTACIÓN DE VALORES DE ÍNDICES FINANCIEROS CON RESULTADOS DE EFICIENCIAS RELATIVAS EN UNA EMPRESA CONSTRUCTORA
}

\author{
CARMEN RESCALA ${ }^{1}$ \\ GUSTAVO DEVICENZI ${ }^{2}$ \\ GRICELA ROHDE ${ }^{1}$ \\ MA. LILIANA BONAFFINI ${ }^{1}$ \\ GUSTAVO BERNAOLA ${ }^{3}$
}

${ }^{1}$ FACULTAD DE CIENCIAS ECONÓMICAS - UNNE ${ }^{2}$ FACULTAD DE INGENIERÍA - UNNE ${ }^{3}$ FACULTAD REGIONAL RESISTENCIA - UTN Los autores agradecen la colaboración prestada por la becaria Rita Alejandra Pavón. 


\title{
RESUMEN
}

$\mathrm{E}$

1 propósito del presente trabajo es realizar un análisis financiero de los Estados Contables de una empresa constructora de la ciudad de Resistencia, mediante el estudio de índices financieros-económicos cuantitativos para luego comparar ese análisis con el efectuado mediante el modelo matemático denominado Análisis Envolvente de Datos (DEA- Data Envelopment Analyst). Los Estados Contables considerados corresponden a seis Ejercicios Económicos y son utilizados para evaluar la marcha del negocio en su proceso de creación de valor. A partir del contraste entre los dos procedimientos empleados se determinan los períodos más eficientes así como las variables de mayor contribución en la ineficiencia de la gestión de administración.

Palabras clave: Empresas constructoras - Análisis financiero - DEA - Eficiencia.

\begin{abstract}
The purpose of this study is to conduct a financial analysis of the Balance Sheet of a construction company in the city of Resistencia, through the study of quantitative economicfinancial indices and then compare that analysis with the results for the mathematical model called Data Envelopment Analysis (DEA). The financial statements taken under six fiscal years are used to evaluate the progress of business in value creation process. From the contrast between the two procedures used is determined the most efficient periods as well as the major contributing variables in the inefficiency of the admi-nistration management.
\end{abstract}

Keywords: Construction companies - Financial analysis - DEA - Efficiency

\section{INTRODUCCIÓN}

La situación financiera, económica y patrimonial de una empresa tiene íntima relación con el contexto en el cual está inserta. El grado de incertidumbre dentro del área de actividad de una organización, depende, entre otras variables, de las condiciones del entorno o macroambiente y del plazo de proyección fijado. Si por ejemplo, el mercado en el que se desarrolla su actividad no opera en forma normal, ello se verá reflejado en la empresa. Por eso, es imprescindible que los directivos se basen en el comportamiento real y posible del contexto.

Las empresas son sistemas que se desarrollan en un medio ambiente y están influenciadas por el entorno, con el que intercambian elementos y generan corrientes de entradas y de salidas de flujos, previo proceso de transformación, para el logro de los objetivos organizacionales. La industria de la construcción es uno de los sectores productivos más importantes 
para el desarrollo de las economías regionales, constituyendo, al mismo tiempo, una actividad intensiva en cuanto a la ocupación de mano de obra en el mercado laboral, en virtud de la gran cantidad de insumos que requiere, posibilitando la participación directa de muchas empresas constructoras. La fuerte dependencia de las condiciones macroeconómicas, tanto en el ámbito nacional como provincial y las características propias de la actividad, con temas puntuales a resolver como la predicción de los costos, la fijación de precios al presupuestar una obra, el valor desconocido de los recursos hasta que la obra se materializa, las características diversas de los trabajos que se realizan, la discontinuidad de la mano de obra en su accionar, con su alta rotación, los gremios con poder, la existencia de una gran cantidad de proveedores, la producción anticíclica, las legislaciones que imponen cláusulas gatillo, entre otras causas, han llevado al sector de la construcción a grandes oscilaciones en su evolución, pasando por períodos de grandes crecimientos, a otros de bruscas depresiones.

El entorno en el cual se desarrolla la empresa es cambiante, incierto y complejo, por esa razón, algunos autores lo clasifican en entorno general y específico. El primero incluye las variables donde se desenvuelven las empresas, como los procesos tecnológicos, políticos, económicos, etc.; el segundo incluye las variables que afectan en forma particular a la empresa, como proveedores, clientes, competidores. Ambos entornos no tienen fronteras nítidas entre sí.

Lo más importante es identificar las variables que afectan en forma positiva o negativa a la organización, sin diferenciar el escenario en que se encuentren (Lammattina, 2006)ํ․․․․

\section{LAS EMPRESAS CONSTRUCTORAS DE RESISTENCIA - CHACO}

En la ciudad de Resistencia, Chaco, la industria de la construcción es una de las actividades más dinámicas en la contribución al crecimiento de su economía, enfrentándose a un mercado competitivo, siendo imperioso para las empresas la necesidad de efectuar un control y evaluación de su eficiencia, para posibilitar una utilización eficaz de los recursos si pretenden mejorar su rentabilidad. Es imprescindible para ellas poner en marcha la creación de valor. Las empresas se vuelven sistemas creadores de valor cuando desarrollan dinámicas productivas y competitivas, conectándose con los mercados, donde se encuentran los recursos necesarios para satisfacer sus necesidades. El concepto de creación de valor está asociado a la eficiencia, ya que las empresas eficientes son las que generan mayor valor empresarial.

\section{ANÁLISIS FINANCIERO DE UNA EMPRESA CONSTRUCTORA}

En el presente trabajo se efectuó, en una primera etapa, el análisis de los Estados Contables de una empresa constructora en particular, a través de la aplicación e interpretación de algunos índices económicos y financieros, con el objeto de determinar el Ejercicio Contable más eficiente de un período de seis Ejercicios. En una segunda instancia, se realizó la aplicación del 
Modelo seleccionado, Análisis Envolvente de Datos -DEA CCR-, orientado a los outputs, con rendimientos constantes a escala utilizando el software Frontier Analyst.

Para este análisis se escogieron las siguientes variables, según el Nivel Jerárquico Superior de la empresa, como las más representativas para medir la eficiencia:

1) variables de entrada: Activo Corriente y Bienes de Uso;

2) variables de salida: Ventas En una tercera etapa, se comparan los resultados obtenidos en las dos instancias anteriores.

\section{PRIMERA ETAPA}

Para efectuar el análisis del objeto de estudio se construyeron cuadros indicadores de la empresa, correspondiente a los seis últimos Balances.

ÍNDICES FINANCIEROS DE LA EMPRESA - PERÍODO 2004-20O9

\begin{tabular}{|l|c|c|c|c|c|c|}
\hline PERÍODO & 2.004 & 2.005 & 2.006 & 2.007 & 2.008 & 2.009 \\
\hline ÍNDICES & & & & & & \\
\hline Liquidez & 2,56 & 2,33 & 2,15 & 1,76 & 2,02 & 1,78 \\
\hline Liquidez Seca (-stock) & 2,56 & 2,33 & 2,15 & 1,57 & 1,64 & 1,78 \\
\hline Prueba Acida (-stock - CxC) & 1,69 & 1,78 & 1,67 & 1,28 & 0,83 & 0,50 \\
\hline Período medio cobranzas & 16,10 & 11,94 & 21,05 & 8,81 & 29,77 & 54,73 \\
\hline Período medio pagos & 39,79 & 19,08 & 23,99 & 40,97 & 63,00 & 59,36 \\
\hline EBITDA (U.T.+Int.+Imp+Amort) & 127,00 & 175,00 & 422,00 & 634,00 & 547,00 & $1.002,00$ \\
\hline Rent s/ Ventas & 0,09 & 0,06 & 0,15 & 0,11 & 0,08 & 0,09 \\
\hline Rent s/ Pat (ROE) & 0,37 & 0,30 & 0,67 & 0,75 & 0,46 & 0,52 \\
\hline Endeudamiento & 0,28 & 0,44 & 0,59 & 0,60 & 0,56 & 0,70 \\
\hline Solvencia & 4,53 & 3,28 & 2,70 & 2,67 & 2,80 & 2,43 \\
\hline Partic Cap Ajeno & & & & 0,19 & 0,15 & 0,35 \\
\hline Du Pont & 0,31 & 0,21 & 0,42 & 0,48 & 0,31 & 0,35 \\
\hline Leverage & 1,17 & 1,43 & 1,58 & 1,56 & 1,46 & 1,50 \\
\hline
\end{tabular}

De los indicadores calculados se trabajó con Liquidez Ácida, Endeudamiento y Solvencia, teniendo estos ratios una adecuada representatividad de los aspectos económicos y financieros de la empresa, de acuerdo a información suministrada por la Gerencia. 
CUADRO DE ÍNDICES COMPARATIVOS DE LA EMPRESA

\begin{tabular}{|c|c|c|c|c|}
\hline \multirow{2}{*}{ EJERCICIO } & \multicolumn{4}{|c|}{ INPUTS } \\
\hline & LIQUIDEZ ÁCIDA & ENDEUDAMIENTO & SOLVENCIA & VENTAS \\
\hline $\mathbf{2 0 0 4}$ & 2.55 & 0,28 & 4,53 & $\$ 1.156 .512,643,28$ \\
\hline $\mathbf{2 0 0 5}$ & 2.33 & 0,44 & 3,28 & $\$ 1.680 .620,58$ \\
\hline $\mathbf{2 0 0 6}$ & 2.15 & 0,59 & 2,70 & $\$ 2.253 .657,49$ \\
\hline $\mathbf{2 0 0 7}$ & 1.57 & 0,60 & 2,67 & $\$ 5.096 .966,68$ \\
\hline $\mathbf{2 0 0 8}$ & 1.64 & 0,56 & 2,80 & $\$ 5.333 .197,60$ \\
\hline $\mathbf{2 0 0 9}$ & 1.78 & 0,70 & 2,43 & $\$ 8.368 .680,49$ \\
\hline
\end{tabular}

\section{ANÁLISIS DE LOS ESTADOS CONTABLES ÍNDICE DE LIQUIDEZ ÁCIDA:}

La prueba ácida es uno de los indicadores financieros utilizados para medir la liquidez de una empresa.

Uno de los elementos más importantes de la estructura de la empresa es la disponibilidad de recursos para cubrir los pasivos a corto plazo, debido a que le permite garantizar que en una eventualidad se disponga de recursos sin tener que recurrir a financiamiento adicional de terceros, precisamente para cubrir un pasivo.

Para determinar la disponibilidad de recursos que posee la empresa para la cobertura de los pasivos a corto plazo, se recurre a la prueba ácida, como indicador de la capacidad de pago de la empresa sin la necesidad de realizar sus inventarios.

La disponibilidad de efectivo o bienes y derechos fácilmente convertibles en efectivo de la empresa está representada por Caja y Bancos, las inversiones a corto plazo, las cuentas por cobrar corrientes (cuentas por cobrar por Ventas y otros créditos) y los inventarios.

La prueba ácida excluye los inventarios, por lo que solo se tiene en cuenta la cartera de clientes, el efectivo y algunas inversiones. La razón por la que se excluyen los inventarios, es porque se supone que la empresa no debe estar supeditada a la venta de sus inventarios para poder pagar las deudas. Esto es especialmente importante en aquellas en la que sus inventarios no son de fácil venta o en empresas en decaimiento comercial.

FÓRMULA PARA CALCULAR LA PRUEBA ÁCIDA: PA= $\frac{\text { Activo Corriente-Inventarios }}{\text { Pasivo Corriente }}$

En el caso que nos ocupa, si comparamos los valores obtenidos en los seis años, éstos indican para el año 2004 (ver Cuadro de Índices Comparativos) la más alta cifra de disponibilidad para hacer frente a las deudas a corto plazo. En ese período el índice de liquidez ácida de 2.55 indica que por cada peso que debe la empresa, posee \$2.55 para pagarlo.

Se supone que el resultado ideal sería la relación 1:1, un peso que se debe y un peso que 
se tiene para pagar, esto garantizaría el pago de la deuda a corto plazo y llenaría de confianza a cualquier acreedor (Biondi, 2003) 2 .

\section{ÍNDICE DE ENDEUDAMIENTO:}

Uno de los ratios o índices analizados es el conocido como razón de endeudamiento, que se determina a través del siguiente cociente:

$$
\text { Endeudamiento }=\frac{\text { Pasivo }}{\text { Patrimonio Neto }}
$$

Por endeudamiento entendemos la participación que tienen los capitales de terceros en la mezcla de aportes de recursos a la empresa que se considera.

Las empresas recurren a distintas combinaciones para obtener fondos, analizando y eligiendo la forma más conveniente.

La razón de endeudamiento establece por cada peso de recursos aportados por el propietario, cuánto han aportado los acreedores.

La comparación de este ratio, para la misma empresa en distintos períodos, nos da una visión mejorada del índice. En este caso, los cocientes arrojados son: 0.28; 0.44; 0.59; 0.60; 0.56; 0.70 para los períodos analizados.

Esto significa, por ejemplo, para el año 2009, con un índice de 0.70, (976/ 1395) que por cada peso del Patrimonio Neto, los terceros aportaron a la sociedad (acreedores) \$0.70. En la medida en que este indicador se incremente, significará que los acreedores están asumiendo más riesgo.

Estos valores ponen en evidencia que en el período 2009 los acreedores han tenido una mayor participación en la financiación de los recursos totales que en los períodos anteriores (Pérez, 2005), lo cual para las empresas constructoras es un indicador de que, dada la trayectoria de las mismas, se han podido incrementar los créditos obtenidos para realizar inversiones, utilizando el capital disponible y evitando la tenencia de capital ocioso.

\section{INDICE DE SOLVENCIA:}

Este índice es una razón financiera de liquidez general, que está formado por el siguiente cociente: Cuanto mayor sea el valor de esta razón financiera, mayor será la capacidad de la empresa de pagar sus deudas. Este índice de solvencia puede arrojar diferentes resultados:

\begin{tabular}{|l|l|l|}
\hline$>1$ & $\mathrm{~A}>\mathrm{P}$ & Favorable \\
\hline$=1$ & $\mathrm{~A}=\mathrm{P}$ & Límite de la garantía \\
\hline$<1$ & $\mathrm{~A}<\mathrm{P}$ & Desfavorable, quiebra técnica \\
\hline \multicolumn{3}{|c|}{} \\
İNDICE DE SOLVENCIA (Lammattina, 2006)
\end{tabular}

Si se observa el Cuadro de Índices Comparativos expuesto precedentemente, todos los períodos resultan favorables, por ser los resultados mayores que la unidad, no obstante el período de mayor solvencia corresponde al año 2004, con un índice de 4.53. 
En la segunda etapa, se realizó la aplicación del Modelo seleccionado DEA CCR utilizando el software Frontier Analyst.

El problema de Programación Lineal ${ }^{a}$ planteado para este modelo, que considera rendimientos constantes a escala en el conjunto de producción, se delineó de la siguiente manera:

$$
M I N_{u, v} \quad W_{0}=\sum_{r=1}^{s} u_{i} x_{i 0}
$$

Las restricciones del problema quedaron definidas de la siguiente manera:

$$
\text { 1) } \sum_{i=1}^{m} u_{i} x_{i j} \geq \sum_{r=1}^{s} v_{r} y_{r j} \quad \text { con } j=1,2, \ldots, n \text { o bien } \sum_{i=1}^{m} u_{i} x_{i j}-\sum_{r=1}^{s} v_{r} y_{r j} \geq 0
$$

2) La suma de los productos ponderados es igual a 1(restricción de normalización).

$$
\sum_{r=1}^{s} u_{r} y_{r 0}=1
$$

3) Las variables de decisión (los pesos) son no negativas: $u_{i} ; v_{i} \geq \xi>0$

Es necesario destacar que se utilizaron dos inputs ${ }^{\mathrm{b}}$ y un output ${ }^{\mathrm{c}}$ para poder realizar los gráficos en el plano, representando en ellos la frontera y los puntos que indican la eficiencia de la empresa considerada en los distintos períodos contables.

A continuación figuran los datos de las variables seleccionadas por la Gerencia de la Empresa correspondiente a los años analizados.

VARIABLES DE ENTRADA Y DE SALIDA

\begin{tabular}{|c|c|c|c|}
\hline Unidad & Activo Cte (Input) & Bs Uso (Input) & Ventas (Output) \\
\hline 2004 & 151 & 197 & 1.156 \\
\hline 2005 & 231 & 268 & 1.681 \\
\hline 2006 & 581 & 235 & 2.254 \\
\hline 2007 & 741 & 409 & 5.097 \\
\hline 2008 & 1.085 & 418 & 5.333 \\
\hline 2009 & 1.739 & 631 & 8.369 \\
\hline
\end{tabular}

Utilizando los datos de la tabla anterior se plantearon los problemas de programación lineal (uno por cada ejercicio o unidad de análisis) empleando el software Frontier Analyst y se obtuvieron los siguientes resultados:

\footnotetext{
a Programación Lineal: es una de las principales ramas de la Investigación Operativa. En esta categoría se consideran todos aquellos modelos de optimización donde las funciones que lo componen, es decir, función objetivo y restricciones, son funciones lineales de las variables de decisión.

${ }^{\mathrm{b}}$ Inputs: variables de entrada para un proceso de producción.

c Output: variable de salida para un proceso de producción.
} 


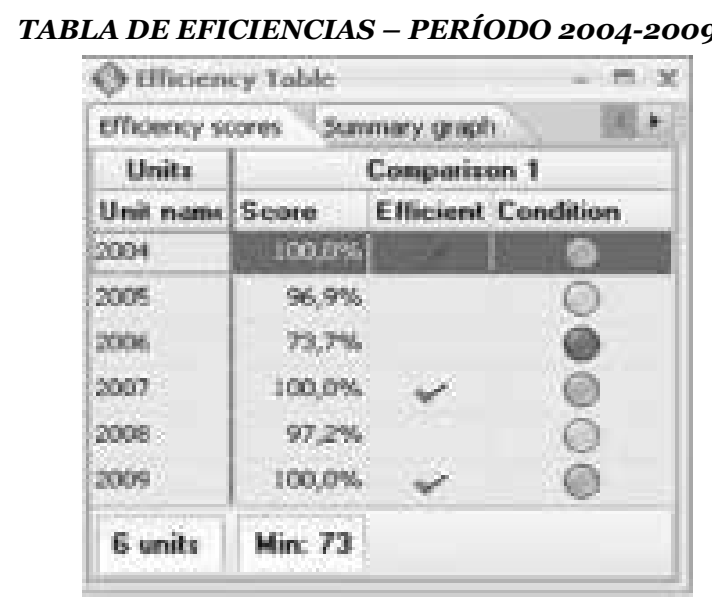

En el análisis de los 6 ejercicios contables de la Empresa seleccionada y siendo ventas, la variable de salida (output), se observan tres períodos (2004, 2007 y 2009) con una eficiencia relativa del $100 \%$.

A continuación se muestra la Frontera de Eficiencia, los puntos sobre la misma representan los mejores resultados alcanzables considerando el conjunto de mediciones realizadas, es decir representan los períodos eficientes. Los puntos fuera de la frontera indican los ciclos evaluados como ineficientes.

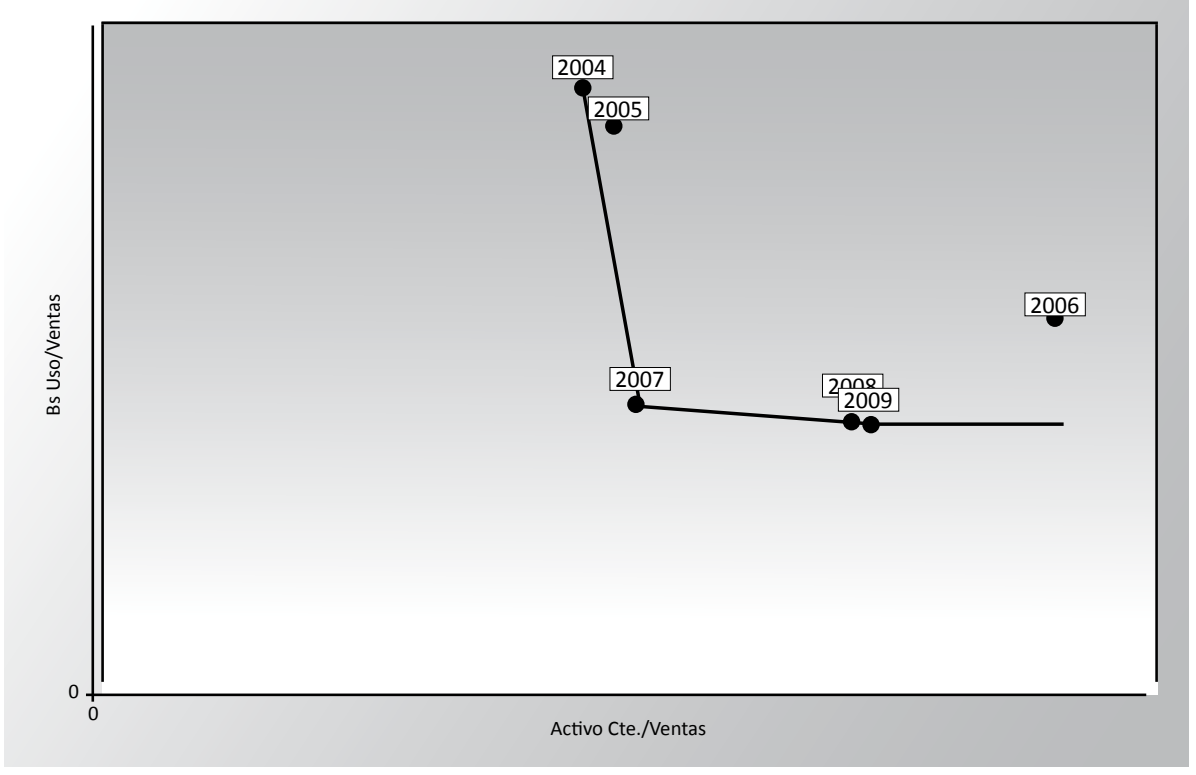

GRAFICO DE LA FRONTERA DE EFICIENCIA

Fuente: (Rescala, Devicenzi, Rohde, Bonaffini, Bernaola, Giraudo y Martínez,201o) 
Continuando el análisis, se determinó qué deben hacer el resto de las unidades consideradas ineficientes para alcanzar la frontera y convertirse en unidades $100 \%$ eficientes.

Se analizaron cada una de las unidades ineficientes:

\section{AÑO 2005}

El conjunto de referencia en esta unidad está dada por las unidades eficientes 2004 y 2007. En el siguiente gráfico se puede observar cómo confronta el comportamiento de esta unidad con respecto al conjunto de referencia con el fin de establecer mejoras competitivas y mejores prácticas. Como existen dos unidades en el conjunto de referencia, también debemos determinar la importancia de cada una.

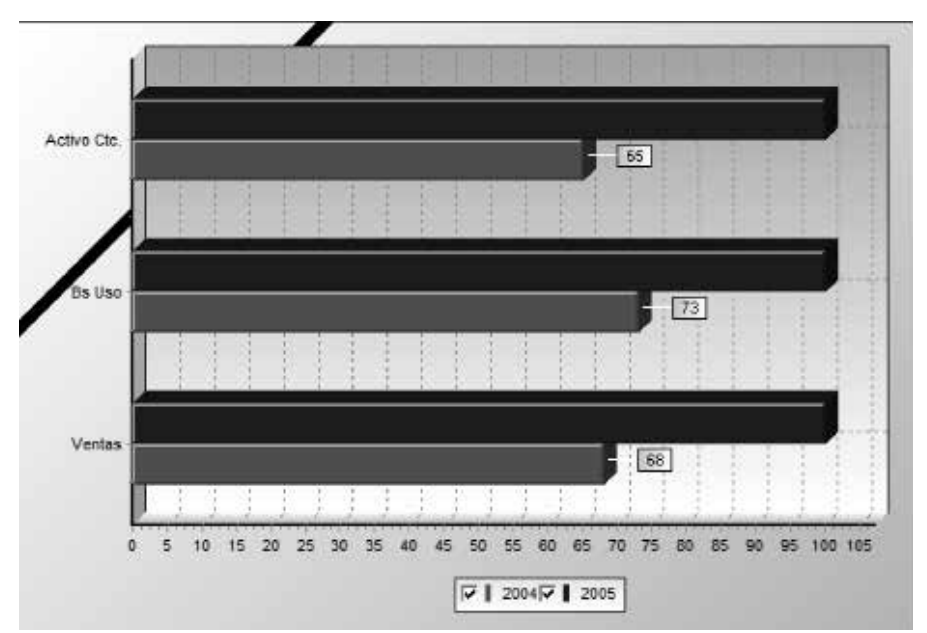

GRÁFICO DE BARRAS COMPARATIVO DE LA UNIDAD INEFICIENTE 2005 CON LA UNIDAD EFICIENTE 2004

Las primeras barras que se utilizan en cada variable representan a la unidad bajo análisis (2005) y las segundas barras representan a una de las unidades eficientes, en este caso, el 2004. El gráfico representa que en el año 2004 con un 35\% menos de Activo Corriente y un 27\% menos de Bienes de Uso, se logro un 68\% de las Ventas del 2005.

Se compara a continuación la unidad en análisis con la otra unidad de referencia, el 2007:

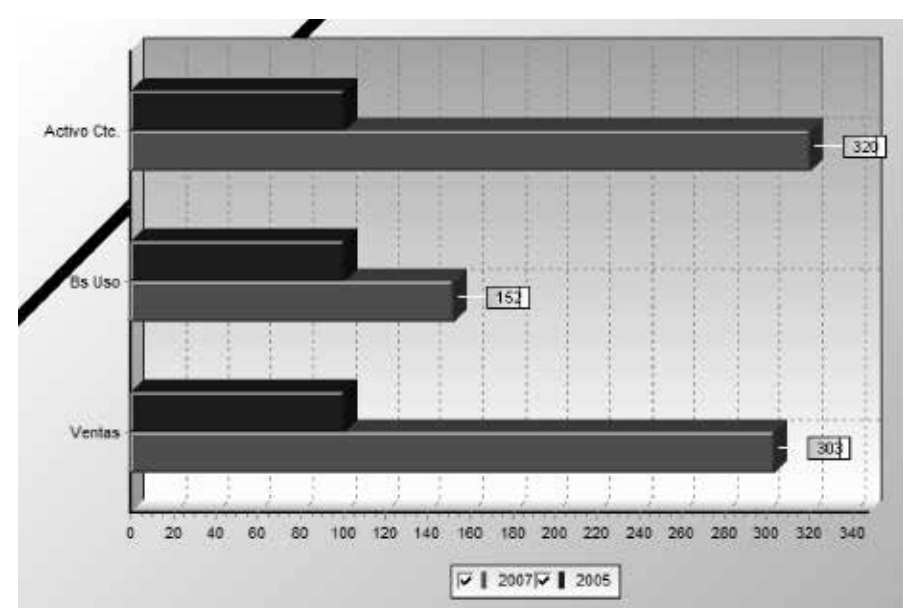


En este caso, podemos decir que en el año 2007, con un 220\% más de Activo Corriente y un 52\% más de Bienes de Uso, se logró un 203\% más de Ventas que en el 2005.

En los informes emitidos por el software, los datos arrojan la medida en que cada unidad eficiente contribuye a la ineficiencia de la unidad bajo análisis.

\section{CONTRIBUCIONES DE LAS UNIDADES EFICIENTES A LA INEFICIENCIA DE LA UNIDAD 2005}

\begin{tabular}{|c|c|c|}
\hline Unidad de Referencia & Input/Output & Contribuciones \\
\hline $\mathbf{2 0 0 4}$ & Activo Cte (Input) & $80,81 \%$ \\
\hline $\mathbf{2 0 0 4}$ & Bs Uso (Input) & $90,87 \%$ \\
\hline $\mathbf{2 0 0 4}$ & Ventas (Output) & $82,41 \%$ \\
\hline $\mathbf{2 0 0 7}$ & Activo Cte (Input) & $19,19 \%$ \\
\hline $\mathbf{2 0 0 7}$ & Bs Uso (Input) & $9,13 \%$ \\
\hline $\mathbf{2 0 0 7}$ & Ventas (Output) & $17,59 \%$ \\
\hline
\end{tabular}

Los datos plasmados en el cuadro se pueden observar más claramente a través del gráfico siguiente:

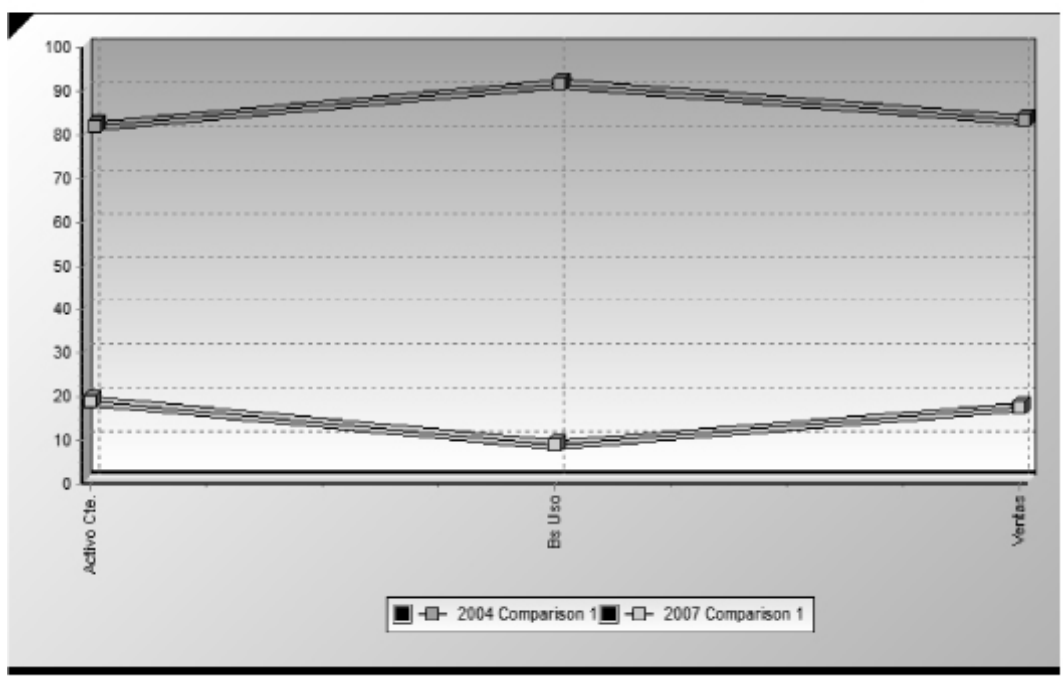

GRÁFICO DE CONTRIBUCIONES DE LAS UNIDADES EFICIENTES A LA INEFICIENCIA DE LA UNIDAD 2005

En el eje " $x$ " se han representados los inputs y los outputs; en el eje "y" la contribución a la ineficiencia de cada unidad perteneciente al conjunto de referencia; la línea superior, el año 2004 y la inferior, el año 2007. La principal utilidad de esta opción es que permite depurar la información aportada por la comparación entre las unidades de referencia y la que se en- 
cuentra bajo análisis. En efecto, si una DMU (Unidad de toma de decisión) contribuye escasamente, no será una buena referencia a la hora de establecer comparaciones. Por otro lado, la identificación de la unidad o unidades que más contribuyen, permite una mayor comprensión de la naturaleza de las ineficiencias que presenta la unidad bajo estudio.

En este caso, la media de la contribución de la unidad 2004 es de 84,65\% y la del 2007, 15,34\%, por lo tanto la que más influye en la ineficiencia de la unidad 2005 es la DMU 2004.

\section{MEJORAS POTENCIALES:}

Se buscó determinar qué debe hacer la unidad 2005 para lograr llegar a la frontera de eficiencia, o lo que es lo mismo, qué mejoras debe realizar para lograr ser una unidad eficiente.

El análisis del programa nos arrojó los valores objetivos para hacer de la unidad 2005 una unidad eficiente de acuerdo con la siguiente fórmula:

Mejora Input $/$ output $=\frac{\text { valor actual }}{\text { valor objetivo }} \cdot 100$

La unidad 2005 sólo debería realizar mejoras en el output ventas para lograr un incremento cercano al 3,15\% y ser una unidad eficiente.

CUADRO DE LA MEJORA POTENCIAL DE LA UNIDAD INEFICIENTE 2005
\begin{tabular}{|c|c|c|}
\hline $\mathbf{2 0 0 5}$ & valor actual & Mejora potencial \\
\hline Activo Cte (Input) & 231 & 231 \\
\hline Bs Uso (Input) & 268 & 268 \\
\hline Ventas (Output) & 1.681 & 1.681 \\
\hline
\end{tabular}

\section{CONTRIBUCIONES INPUTS Y OUTPUTS:}

Determinar para una DMU, qué variables Input/Ouput han sido utilizadas para obtener la puntuación de eficiencia y cuáles han sido ignoradas, permite responder de que manera éstas pueden ayudar a validar la puntuación de eficiencia obtenida.

El DEA optimiza buscando las ponderaciones que más favorecen a la unidad evaluada. Los valores se muestran normalizados como porcentajes en el siguiente gráfico: 


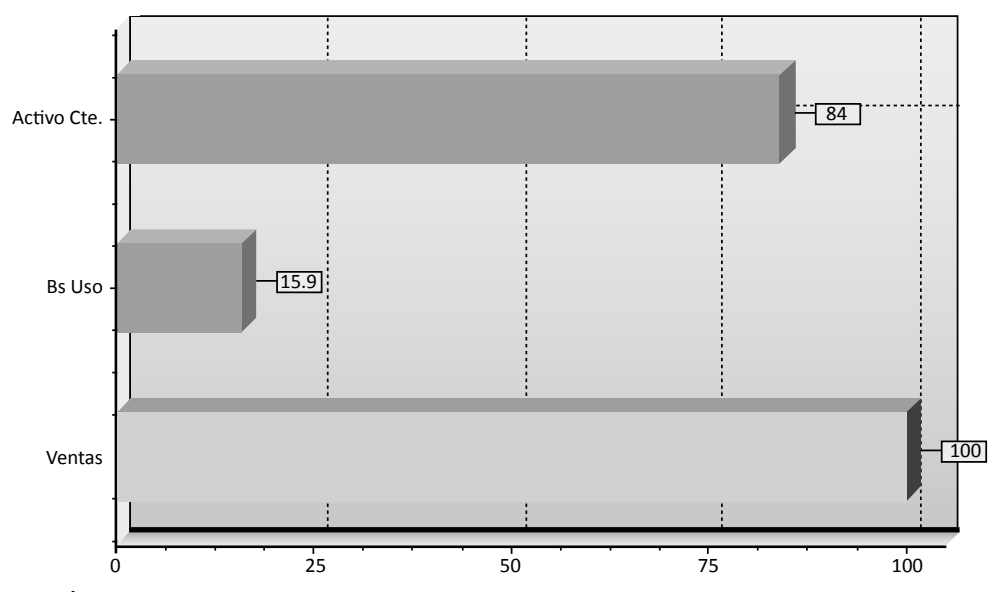

GRÁFICO DE LAS CONTRIBUCIONES DE LOS INPUTS Y DE LOS OUTPUTS

Se observa que el output Ventas es el que mayor énfasis ha tenido en la determinación de la ineficiencia de la unidad 2005 con un 100\%, seguido por el input Activo Corriente $84 \%$ y luego por el input restante Bienes de uso 15,9\%, de muy poca incidencia.

Se procedió de la misma manera para las otras unidades ineficientes y los resultados obtenidos fueron:

\section{AÑO 2006:}

El conjunto de referencia en esta unidad está dado por las unidades eficientes 2007 y 2009. La unidad 2006 es la que más alejada se encuentra de la frontera de eficiencia. Los datos que arrojó el software son los siguientes:

* Los porcentajes de contribución del conjunto de referencia a la ineficiencia de la unidad bajo análisis, son los siguientes:

CONTRIBUCIONES DE LAS UNIDADES EFICIENTES A LA INEFICIENCIA DE LA UNIDAD 2006

\begin{tabular}{|c|c|c|}
\hline Unidad de Referencia & Input/Output & Contribuciones \\
\hline $\mathbf{2 0 0 7}$ & Activo Cte (Input) & $22,01 \%$ \\
\hline $\mathbf{2 0 0 7}$ & Bs Uso (Input) & $30,04 \%$ \\
\hline $\mathbf{2 0 0 7}$ & Ventas (Output) & $28,74 \%$ \\
\hline $\mathbf{2 0 0 9}$ & Activo Cte (Input) & $77,99 \%$ \\
\hline $\mathbf{2 0 0 9}$ & Bs Uso (Input) & $69,96 \%$ \\
\hline $\mathbf{2 0 0 9}$ & Ventas (Output) & $71,26 \%$ \\
\hline
\end{tabular}

* En el año 2007 con respecto al año 2006, se utilizó un 27\% más de Activo Corriente y $74 \%$ más de Bienes de Uso logrando un 126\% más de Ventas.

* La unidad 2009 con respecto a la unidad bajo análisis utilizó un 199\% más de Activo Corriente y un 166\% más de Bienes Uso logrando obtener un 271\% más de Ventas. 
* En cuanto a las mejoras potenciales los resultados arrojan que sólo el output Ventas necesitaría una mejora potencial de 35,77\%, como lo muestra el siguiente cuadro:

CUADRO DE LA MEJORA POTENCIAL DE LA UNIDAD INEFICIENTE 2006

\begin{tabular}{|c|c|c|c|}
\hline 2006 & valor actual & valor objetivo & Mejora Potencial \\
\hline Activo Cte (Input) & 581 & 581 & 0 \\
\hline Bs Uso (Input) & 235 & 235 & 0 \\
\hline Ventas (Output) & 2.254 & 3060,29 & $35,77 \%$ \\
\hline
\end{tabular}

* Con respecto a la contribución de los inputs y outputs, el que mayor peso tuvo a la hora de determinar la ineficiencia de la unidad fue el output Ventas con un 100\%, seguido por el input Bienes de Uso con 83,8\% y por último el input Activo Corriente con un 16,1\%, claramente menor.

\section{AÑO 2008:}

El conjunto de referencia de esta unidad está dado por la unidades 2007 y 2009. Los datos surgidos del estudio fueron:

* El programa analizó cuál fue el porcentaje de las contribuciones de cada unidad a la ineficiencia de la unidad Año 2008:

\section{CONTRIBUCIONES DE LAS UNIDADES EFICIENTES A LA INEFICIENCIA DE LA UNIDAD 2008}

\begin{tabular}{|c|c|c|}
\hline Unidad de Referencia & Input/Output & Contribuciones \\
\hline $\mathbf{2 0 0 7}$ & Activo Cte (Input) & $11,85 \%$ \\
\hline $\mathbf{2 0 0 7}$ & Bs Uso (Input) & $16,97 \%$ \\
\hline $\mathbf{2 0 0 7}$ & Ventas (Output) & $16,11 \%$ \\
\hline $\mathbf{2 0 0 9}$ & Activo Cte (Input) & $88,15 \%$ \\
\hline $\mathbf{2 0 0 9}$ & Bs Uso (Input) & $83,03 \%$ \\
\hline $\mathbf{2 0 0 9}$ & Ventas (Output) & $83,89 \%$ \\
\hline
\end{tabular}

* En el año 2007 con un 32\% menos de Activo Corriente y un 3\% menos de Bienes de Uso se logro un 5\% menos de Ventas o un 95\% de Ventas del 2008.

* En el año 2009, con un 60\% más de Activo Corriente y un 50\% más de Bienes de Uso se logró un 56\% más de Ventas con respecto al año 2008.

* Las mejoras a realizar en este caso son mínimas, el output Ventas debe aumentarse sólo en un 2,89\% para poder alcanzar la frontera de eficiencia, como se muestra en el siguiente cuadro:

CUADRO DE LA MEJORA POTENCIAL DE LA UNIDAD INEFICIENTE 2008

\begin{tabular}{|c|c|c|c|}
\hline $\mathbf{2 0 0 8}$ & valor actual & valor objetivo & Mejora Potencial \\
\hline Activo Cte (Input) & 1.085 & 1.085 & 0 \\
\hline Bs Uso (Input) & 418 & 418 & 0 \\
\hline Ventas (Output) & 5.333 & 5487,14 & $2,89 \%$ \\
\hline
\end{tabular}


* Según los datos, el output Ventas nuevamente tuvo un énfasis del 100\% en la determinación de la ineficiencia de la unidad seguido por Bienes de Uso 83,2\% y luego por Activo Corriente 16,7\%.

\section{ANÁLISIS DE CORRELACIÓN ENTRE LAS VARIABLES}

Los siguientes gráficos representan las relaciones entre Activo Corriente y Ventas en el primero de ellos, mientras que en el segundo la existente entre Bienes de Uso y Ventas:

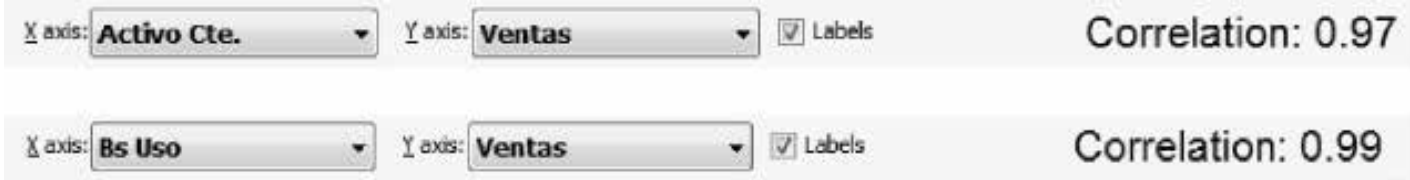

Los dos inputs tienen una relación directa con el output Ventas (dado por el signo positivo del coeficiente de correlación), lo que significa que si alguno de los inputs aumenta también lo hará el output en la medida del coeficiente que corresponda. Por otra parte, aunque los dos inputs tienen alto coeficiente de correlación, podemos decir que Bienes de Uso es ligeramente más relevante que Activo Corriente dado que su coeficiente es de 0.99.

\section{TERCERA ETAPA:}

A continuación se realiza la contrastación del análisis financiero de los resultados de una empresa constructora, con los valores de eficiencias relativas obtenido mediante el modelo análisis de envoltura de datos (DEA). Un ratio financiero, por su forma de calcular, permite destacar ciertos aspectos de la empresa, de manera tal que para obtener una visión global de ella será necesario utilizar un conjunto de numerosos ratios. En el presente trabajo, donde se analizaron los indicadores "Liquidez Ácida", "Endeudamiento" y "Solvencia”, el 2004 arrojó los valores más favorables de los mismos.

Los datos volcados en el Cuadro de Índices Comparativos que se muestran en este trabajo, explican el comportamiento de la situación económico-financiera de la empresa analizada, a modo de diagnóstico de áreas de la misma, pudiendo ser utilizada como herramienta de evaluación. Por ejemplo, ver si el crecimiento de las Ventas es proporcional al crecimiento de la actividad del sector de la construcción en la Provincia en un período, analizar los valores del endeudamiento, su relación con las Cuentas a Cobrar y la evolución de los índices de Liquidez, para poder determinar si los mismos guardan coherencia, como en este caso, en que el aumento del endeudamiento se corresponde con un aumento de las cuentas por cobrar. De esta forma la empresa recurre al crédito para cancelar obligaciones del Pasivo Corriente, principalmente en los gastos correspondientes a sueldos e impuestos y que no se destinan a un aumento de 
la inversión en activo fijo o gastos a recuperar. El método DEA, a diferencia del análisis financiero, utiliza los mejores valores obtenidos por la empresa en los años analizados, los que pueden ser alcanzables si se introducen mejoras de gestión (mejoras potenciales). Por lo tanto el DEA muestra, además de la medida de eficiencia, cómo se pueden mejorar los resultados reduciendo o aumentando proporcionalmente los inputs y/o outputs considerados. A partir de las variables “Activo Corriente", "Bienes de Uso" y "Ventas" se construyó un sistema para la aplicación del modelo DEA en los seis ejercicios, concluyendo:

* El año 2007 tuvo una baja incidencia en la ineficiencia de las DMU 2005, 2006 y 2008.

* El año 2004 tuvo una contribución alta en la ineficiencia de la unidad 2005, lo mismo ocurrió con el año 2009 en la ineficiencia de las unidades 2006 y 2008.

* La contribución del output "Ventas" a la ineficiencia de las unidades bajo análisis es del $100 \%$ porque se trabajó con el modelo DEA orientado a los outputs.

* El año 2006 requiere la mayor mejora potencial, donde el valor arrojado para las ventas es de $36 \%$, mientras que en las otras unidades ineficientes los requerimientos de mejora no llegan al $5 \%$.

* La correlación entre las variables trabajadas es cercana al 100\%, indicando de esta manera la correcta selección de las mismas.

Quienes conforman el nivel ejecutivo de la empresa tienen que reflexionar sobre los resultados obtenidos para realizar un feedback que les permita corregir, con las estrategias apropiadas, aquellos valores de eficiencia que no se encuentran sobre la frontera. En este trabajo se ha aplicado "un" modelo de análisis, quedando abierta la posibilidad de utilizar otros inputs y/o outputs de acuerdo a los criterios y necesidades planteados por el management de las empresas constructoras.

\section{REFERENCIAS BIBLIOGRÁFICAS.}

1.- Lammattina, O.E. (2006). Análisis de Estados Financieros - Un enfoque integral - $1^{\mathrm{O}}$ edición. Buenos Aires: Osmar D. Buyatti - Librería Editorial.

2.- Biondi, M. (2003). Estados Contables. Presentación, Interpretación y Análisis. Buenos Aires: ERREPAR. 
3.- Pérez, J.O. (2005). Análisis de Estados Contables: un enfoque de gestión. Córdoba: EDUCC - Editorial de la Universidad Nacional de Córdoba. República Argentina.

4.- Rescala, C.; Devicenzi, G.; Rohde, G.; Bonaffini, M.L.; Bernaola, G.; Giraudo, M. y Martínez, H. (2010). Utilización de un modelo matemático para la determinación de factores de eficiencia en una empresa constructora de la ciudad de Resistencia. Revista del Instituto de Matemática, 6 (12), 89-98. Facultad de Ingeniería. Universidad Nacional del Nordeste. ISSN 1850-9827.

\section{CURRÍCULUM VITAE}

\section{CARMEN RESCALA.}

Profesora en Matemática, Física y Cosmografía, Contadora Pública y Especialista en Ingeniería Gerencial. Actualmente es Profesora Titular Ordinaria Dedicación Exclusiva de la Cátedra "Matemática I" de la Facultad Ciencias Económicas de la UNNE y Profesora Titular Ordinaria Dedicación Simple de la Cátedra “Ciencias Básicas” de la Facultad de Arquitectura y Urbanismo de la UNNE. Miembro Fundador y Miembro del Consejo Académico del Instituto de Matemática de la Facultad de Ingeniería de la UNNE. Integrante de la Comisión Directiva en el cargo de Prosecretaria de la Asociación Nacional de Docentes de Matemática de Facultades de Ciencias Económicas y Afines. Es Docente Investigadora, Categoría 3.

carmenrescala@yahoo.com.ar

\section{GUSTAVO HORACIO DEVICENZI.}

Ingeniero en Construcciones / Ingeniero Civil. Especialista en docencia universitaria. Profesor Titular con Dedicación Exclusiva de la Cátedra: "Informática" de la Facultad de Ingeniería de la UNNE y Profesor Titular con Dedicación Simple de la Cátedra "Procesamiento de Datos" de la Facultad de Ciencias Económicas de la UNNE. Codirector del Proyecto: "Modelado Virtual 3d de Carreteras Rurales de dos Carriles para Uso en un Simulador de Conducción de Automóviles”. gdevin@ing.unne.edu.ar

\section{GRICELA ALICIA ROHDE.}

Profesora en Matemática y Cosmografía y Especialista en Investigación Educativa. Actualmente es Profesora Adjunta Ordinaria con Dedicación Exclusiva de la Cátedra "Matemática I" y Auxiliar Docente de Primera de la Cátedra "Matemática II" de la Facultad de Ciencias Económicas de la UNNE. Es Docente Inves-tigadora, Categoría 4.

grohde@eco.unne.edu.ar 


\section{MARÍA LILIANA BONAFFINI.}

Contadora Pública. Profesora con Mayor Dedicación en el cargo de Auxiliar Docente de $1^{\circ}$ Categoría en la Cátedra de Costos de la Facultad de Ciencias Económicas de la UNNE. Coordinadora General del Instituto de Contabilidad de Costos y de Gestión de la Facultad de Ciencias Económicas. Es Docente Investigadora: Categorización 5. Idioma: Inglés Técnico. Primer Nivel. F.C.E. -UNNE. mbonaffini@eco.unne.edu.ar

\section{GUSTAVO BERNAOLA.}

Ingeniero Electromecánico - Especialista en Ingeniería en Calidad. Jefe de Trabajos Prácticos de Organización Industrial Quinto año Ingeniería Electromecánica UTN FRRe. Adjunto Calidad y productividad Cuarto año Ingeniería Electromecánica UTN FRRe. Jefe de Trabajos Prácticos Análisis Matemático II Ingeniería Electromecánica UTN FRRe. Presidente de la Fundación Intecnor Incubadora de Empresas de Base Tecnológica de la UTN FRRe.

gustavo@edesycc.com.ar

Los autores integran el Proyecto acreditado por la Secretaría General de Ciencia y Técnica de la UNNE MOO1/201O, cuyo título es: MODELOS MATEMÁTICOS Y ESTADÍSTICOS PARA LA GESTIÓN DE ADMINISTRACIÓN DE EMPRESAS CONSTRUCTORAS (Segunda Etapa), siendo Directora del mismo la Prof. Carmen Rescala y Co-Director, el Ing. Gustavo Devicenzi. 